Publ. Math. Debrecen

79/1-2 (2011), 231-250

DOI: 10.5486/PMD.2011.500

\title{
Descending maps between slashed tangent bundles
}

\author{
By IOAN BUCATARU (Iasi) and MATIAS F. DAHL (Helsinki)
}

\begin{abstract}
Suppose $T M \backslash\{0\}$ and $T \widetilde{M} \backslash\{0\}$ are slashed tangent bundles of smooth manifolds $M$ and $\widetilde{M}$, respectively. In this paper we first give a differential-topological characterization of those diffeomorphisms $F: T M \backslash\{0\} \rightarrow T \widetilde{M} \backslash\{0\}$ that can be written as $F=\left.(D \phi)\right|_{T M \backslash\{0\}}$ for a diffeomorphism $\phi: M \rightarrow \widetilde{M}$. When this is the case we say that $F$ descends. Using the characterization we obtain two geometric results. First, if $M$ is equipped with two sprays, we obtain sufficient conditions that imply that $F$ descends to a totally geodesic map. Second, if $M$ has two Riemann metrics, we obtain sufficient conditions for $F$ to descend to an isometry.
\end{abstract}

\section{Introduction}

In this paper we study the following differential-topological problem:

(*) Suppose $M$ and $\widetilde{M}$ are smooth manifolds, and suppose that $F$ is a diffeomorphism between slashed tangent bundles

$$
F: T M \backslash\{0\} \rightarrow T \widetilde{M} \backslash\{0\} .
$$

Characterize those maps $F$ that can be written as $F=\left.(D \phi)\right|_{T M \backslash\{0\}}$ for a diffeomorphism $\phi: M \rightarrow \widetilde{M}$, where $D \phi$ is the tangent map of $\phi$. When $F=\left.(D \phi)\right|_{T M \backslash\{0\}}$ one say that map $F$ descends to a map $\phi: M \rightarrow \widetilde{M}[8]$.

Mathematics Subject Classification: Primary: 53C20; Secondary: 53C24, 53C22, 53B30, 53C05, 53C60, 57R50.

Key words and phrases: diffeomorphisms, tangent map, second tangent bundle, geodesic spray, canonical involution, Riemann metric.

I.B. has been supported by grant ID 398 from the Romanian Ministry of Education. M.D. has been supported by Academy of Finland Centre of Excellence programme 213476, the Institute of Mathematics at the Helsinki University of Technology, and Tekes project MASIT03 - Inverse Problems and Reliability of Models. 
Problem $(*)$ is related to anisotropic boundary rigidity problems on Riemannian manifolds [8], [27], [23]. It can also be seen as a differential-topological component of the geodesic conjugacy problem in Riemann geometry, which asks the following: If $F: T M \backslash\{0\} \rightarrow T \widetilde{M} \backslash\{0\}$ maps integral curves of one Riemann metric into integral curves of another Riemann metric, what additional assumptions are required for $F$ to be induced by an isometry. For an overview, see [2].

Let us first note that if $f$ is a diffeomorphism between (unslashed) cotangent bundles

$$
f: T^{*} M \rightarrow T^{*} \widetilde{M}
$$

the analogous problem is well understood. Namely, $f$ descends into a diffeomorphism $\psi: \widetilde{M} \rightarrow M$ if and only if $f$ preserves the canonical 1-forms on $T^{*} M$ and $T^{*} \widetilde{M}$, respectively. This result characterizes diffeomorphic symplectomorphisms between cotangent bundles that arise from diffeomorphisms between the base manifolds. The result can be seen as a consequence of Euler's theorem for homogeneous functions. Alternatively, $f$ defines a map between $M$ and $\widetilde{M}$ since $f$ maps zero covectors to zero covectors [1], [11], [17], [21], [9]. When $f$ is only defined between slashed cotangent bundles this characterization is no longer valid [29].

In this work we study maps $F$ as in equation (1). Hence $F$ is defined and smooth only for non-zero vectors. In this case the problem is more difficult since we can not use the zero section to define a map $\phi: M \rightarrow \widetilde{M}$. We can neither use Euler's theorem for homogeneous functions to deduce that $F$ is linear in the fibre variable. Our first main result is Theorem 3.1. It states that if $F$ is a diffeomorphism $F: T M \backslash\{0\} \rightarrow T \widetilde{M} \backslash\{0\}$, then $F=\left.(D \phi)\right|_{T M \backslash\{0\}}$ for a diffeomorphism $\phi: M \rightarrow \widetilde{M}$ if and only if

$$
D F=\widetilde{\kappa}_{2} \circ D F \circ \kappa_{2},
$$

where $\kappa_{2}$ and $\widetilde{\kappa}_{2}$ are the canonical involutions on $T T M$ and $T T \widetilde{M}$ (Section 2.2). One can interpret Theorem 3.1 as an analogue to Poincaré's lemma for diffeomorphisms; if the derivative of diffeomorphism $F$ satisfies algebraic condition (2), then $F$ can be written as the derivative of another diffeomorphism. This gives a solution to Problem $(*)$.

As an application of Theorem 3.1 we prove Theorems 6.2 and 7.3, which give sufficient geometric conditions for a map to descend. Assuming that $M$ has two sprays, Theorem 6.2 gives sufficient conditions that imply that $F$ descends to a totally geodesic map $\phi: M \rightarrow M$ (Section 5.2). Assuming further that the sprays are induced by two Riemann metrics, we obtain Theorem 7.3, which gives sufficient conditions that imply that $F$ descends to an isometry $\phi: M \rightarrow M$. 
There are two key assumptions in both theorems. The first key assumption is that $F$ maps non-zero Jacobi fields into non-zero Jacobi fields. This means that both theorems essentially describe to what extent Jacobi fields determine the spray (or Riemann metric) [18]. Let us point out that Jacobi fields and curvature are related. However, they are also different, since the covariant derivative is needed to relate one to the other. For results on the relation between curvature and the Riemann metric, see [14], [31], and the Cartan-Ambrose-Hicks theorem [7]. For the real-analytic case, see also [13] and [22]. The second key assumption is that one of the Riemann metrics in Theorem 7.3, say $g$, (or one of the sprays in Theorem 6.2) has a trapping hypersurface $\Sigma \subset M$, that is, a hypersurface that intersects all geodesics of $g$. The existence of such a surface imposes a global restriction on the behaviour of geodesics. For example, there can be no closed geodesics that do no intersect $\Sigma$. This concept is analogous to non-trapping manifolds with boundary [10], which is a natural setting when studying boundary rigidity problems.

Lastly we note that if $F$ satisfies the assumptions in Theorem 7.3, then $F$ necessarily maps integral curves into integral curves (see Step 1 in the proof of Theorem 6.2). Hence Theorem 7.3 is also a contribution to understanding the geodesic conjugacy problem. We emphasize that in Theorem 7.3, there are no local assumptions on curvature, and the base manifold $M$ can have any dimension $\operatorname{dim} M \geq 2$.

\section{Preliminaries}

By a manifold $M$ we mean a topological Hausdorff space with countable base that is locally homeomorphic to $\mathbb{R}^{n}$ with $C^{\infty}$-smooth transition maps and $n=\operatorname{dim} M \geq 1$. All objects are assumed to be $C^{\infty}$-smooth where defined.

The next sections collect results about iterated tangent bundles we will need. For a more detailed discussion and references we refer to [4], [5].

2.1. Iterated tangent bundles. If $M$ is a manifold, let $T M$ be the tangent bundle of $M$. For $r \geq 0$, the $r$ th iterated tangent bundle $T^{r} M$ is defined inductively by setting $T^{r} M=M$ when $r=0$, and $T^{r} M=T\left(T^{r-1} M\right)$ when $r \geq 1$. Let $\pi_{r}$ be the canonical projection operators $\pi_{r}: T^{r+1} M \rightarrow T^{r} M$ when $r \geq 0$. Occasionally we also write $\pi_{T T M \rightarrow M}, \pi_{T M \rightarrow M}, \ldots$ instead of $\pi_{0} \circ \pi_{1}, \pi_{0}, \ldots$ Unless otherwise specified, we always use canonical local coordinates (induced by local coordinates on $M$ ) for iterated tangent bundles. If $x^{i}$ are local coordinates for $M$, we denote induced local coordinates for $T M$, TTM, and TTTM by 
$(x, y),(x, y, X, Y)$ and $(x, y, X, Y, u, v, U, V)$. As above, we usually leave out indices for local coordinates and write $(x, y)$ instead of $\left(x^{i}, y^{i}\right)$. For $p \in M$ let $T_{p} M=\pi_{0}^{-1}(p)$.

For $r \geq 1$, we treat $T^{r} M$ as a vector bundle over the manifold $T^{r-1} M$ with the vector space structure induced by projection $\pi_{r-1}: T^{r} M \rightarrow T^{r-1} M$. Thus, if $\left\{x^{i}: i=1, \ldots, 2^{r-1} n\right\}$ are local coordinates for $T^{r-1} M$, and $(x, y)$ are local coordinates for $T^{r} M$, then vector addition and scalar multiplication are given by

$$
\begin{aligned}
(x, y)+(x, \widetilde{y}) & =(x, y+\widetilde{y}), \\
\lambda \cdot(x, y) & =(x, \lambda y) .
\end{aligned}
$$

For $r \geq 0$, a vector field on an open set $B \subset T^{r} M$ is a smooth map $X: B \rightarrow$ $T^{r+1} M$ such that $\pi_{r} \circ X=\mathrm{id}_{B}$. The set of all vector fields on $B$ is denoted by $\mathfrak{X}(B)$. Suppose that $\gamma$ is a smooth map $\gamma:(-\varepsilon, \varepsilon)^{k} \rightarrow T^{r} M$ where $k \geq 1$ and $r \geq 0$. If $\gamma\left(t^{1}, \ldots, t^{k}\right)=\left(z^{i}\left(t^{1}, \ldots, t^{k}\right)\right)$ in local coordinates $\left(z^{i}\right)$ for $T^{r} M$, then the derivative of $\gamma$ with respect to variable $t^{j}$ is the map $\partial_{t^{j}} \gamma:(-\varepsilon, \varepsilon)^{k} \rightarrow T^{r+1} M$ defined by $\partial_{t^{j}} \gamma=\left(z^{i}, \partial z^{i} / \partial t^{j}\right)$. When $k=1$ we also write $\gamma^{\prime}=\partial_{t} \gamma$ and say that $\gamma^{\prime}$ is the tangent of $\gamma$. If $f: T^{r} M \rightarrow T^{s} \widetilde{M}(r, s \geq 0)$ is a map between iterated tangent bundles

$$
(f \circ c)^{\prime}(t)=D f \circ c^{\prime}(t), \quad t \in I .
$$

Unless otherwise stated we always assume that $I$ is an open interval in $\mathbb{R}$ (and we do not exclude unbounded intervals).

If $\xi \in T^{r} M$ for $r \geq 2$, then there exists a map $V:(-\varepsilon, \varepsilon)^{2} \rightarrow T^{r-2} M$ such that

$$
\xi=\left.\partial_{t} \partial_{s} V(t, s)\right|_{t=s=0} .
$$

2.2. Canonical involution. On the iterated tangent bundle $T^{r} M$ where $r \geq 2$ the canonical involution is the unique diffeomorphism $\kappa_{r}: T^{r} M \rightarrow T^{r} M$ such that

$$
\partial_{s} \partial_{t} c(t, s)=\kappa_{r} \circ \partial_{t} \partial_{s} c(t, s)
$$

for all smooth maps $c:(-\varepsilon, \varepsilon)^{2} \rightarrow T^{r-2} M$. Let also $\kappa_{1}=\operatorname{id}_{T M}$. In local coordinates for TTM and TTTM, it follows that

$$
\begin{aligned}
\kappa_{2}(x, y, X, Y) & =(x, X, y, Y), \\
\kappa_{3}(x, y, X, Y, u, v, U, V) & =(x, y, u, v, X, Y, U, V) .
\end{aligned}
$$

For any $r \geq 1$, we have

$$
\begin{array}{r}
\pi_{r} \circ \kappa_{r+1}=D \pi_{r-1}, \\
\pi_{r-1} \circ \pi_{r} \circ \kappa_{r+1}=\pi_{r-1} \circ \pi_{r} .
\end{array}
$$


If $\phi$ is a map $\phi: M \rightarrow \widetilde{M}$, then equations (5), (6), and (7) imply that

$$
\widetilde{\kappa}_{2} \circ D D \phi \circ \kappa_{2}=D D \phi
$$

As in equation (10) we denote involution operators on $T^{r} M$ and $T^{r} \widetilde{M}$ by $\kappa_{r}$ and $\widetilde{\kappa}_{r}$, respectively. Similarly, we denote projection operators by $\pi_{r}$ and $\widetilde{\pi}_{r}$.

2.3. Slashed tangent bundles. The slashed tangent bundle for $M$ is defined as the open set of non-zero vectors,

$$
T M \backslash\{0\}=\{\xi \in T M: \xi \neq 0\} .
$$

For $r \geq 2$ we generalize and define

$$
T^{r} M \backslash\{0\}=\left\{\xi \in T^{r} M:\left(D \pi_{T^{r-1} M \rightarrow M}\right)(\xi) \in T M \backslash\{0\}\right\} .
$$

When $r \geq 2, \kappa_{r}$ restricts to a diffeomorphism

$$
\kappa_{r}: T^{r} M \backslash\{0\} \rightarrow T\left(T^{r-1} M \backslash\{0\}\right) .
$$

If $F$ is a map $F: T M \backslash\{0\} \rightarrow T \widetilde{M} \backslash\{0\}$, then

$$
\widetilde{\kappa}_{3} \circ D D F \circ \kappa_{3}=D D F \quad \text { on } T T(T M \backslash\{0\}) .
$$

\section{A differential-topological characterization}

Theorem 3.1 below is the first main result in this paper. The theorem is a differential-topological characterization of descending maps between slashed tangent bundles.

Theorem 3.1. Suppose $M$ and $\widetilde{M}$ are smooth manifolds. If $F$ is a smooth map $F: T M \backslash\{0\} \rightarrow T \widetilde{M} \backslash\{0\}$, then the following conditions are equivalent:

(i) There exists a smooth map $\phi: M \rightarrow \widetilde{M}$ such that

$$
F=\left.(D \phi)\right|_{T M \backslash\{0\}}
$$

(ii) On $T T M \backslash\{0\} \cap T(T M \backslash\{0\})$,

$$
D F=\widetilde{\kappa}_{2} \circ D F \circ \kappa_{2}
$$

What is more, if $F$ is a diffeomorphism, and $\phi$ exists, then $\phi$ is a diffeomorphism. 
Let us make three remarks about Theorem 3.1 assuming that $\phi$ exists. First, when $\phi$ exists, it is unique, and the following diagram commutes:

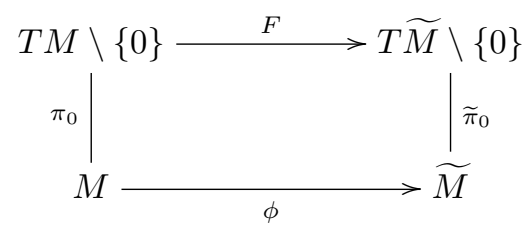

Second, since $F$ is a map between slashed tangent bundles, $\phi$ is necessarily an immersion. Thus, if $\operatorname{dim} M=\operatorname{dim} \widetilde{M}$, the inverse function theorem implies that $\phi$ is a local diffeomorphism. Third, if $\phi$ is a diffeomorphism, then equation $F=D \phi$ extends $F$ into a (smooth) diffeomorphism $F: T M \rightarrow T \widetilde{M}$.

Theorem 3.1 is a direct consequence of the next two lemmas; implication (ii) $\Rightarrow$ (i) follows by Lemma 3.2, the last claim follows by Lemma 3.3, and the easy implication (i) $\Rightarrow$ (ii) follows by equation (10).

Lemma 3.2. Let $F$ be a smooth map $F: T M \backslash\{0\} \rightarrow T \widetilde{M} \backslash\{0\}$ that satisfies condition (ii) in Theorem 3.1, and let $\phi$ be the set-valued map $\phi: M \rightarrow P(\widetilde{M})$,

$$
\phi(p)=\tilde{\pi}_{0} \circ F\left(T_{p} M \backslash\{0\}\right), \quad p \in M,
$$

where $P(\widetilde{M})$ is the power set of $\widetilde{M}$. Then

(i) $\phi$ defines a smooth single-valued map $\phi: M \rightarrow \widetilde{M}$,

(ii) $F=\left.(D \phi)\right|_{T M \backslash\{0\}}$.

Proof. To show that $\phi$ is single-valued we show that map $C: T_{p} M \backslash\{0\} \rightarrow \widetilde{M}$

$$
C(\xi)=\widetilde{\pi}_{0} \circ F(\xi), \quad \xi \in T_{p} M \backslash\{0\},
$$

is constant when $p \in M$ is fixed. If $\xi, \eta \in T_{p} M \backslash\{0\}$ we can find a $w \in T T M \backslash$ $\{0\} \cap T(T M \backslash\{0\})$ such that $\pi_{1}(w)=\xi$ and $D \pi_{0}(w)=\eta$. Using equations (8) and (9), and the assumption on $D F$ we have

$$
\begin{aligned}
C(\xi) & =\tilde{\pi}_{0} \circ F \circ \pi_{1}(w)=\tilde{\pi}_{0} \circ \tilde{\pi}_{1} \circ D F(w)=\tilde{\pi}_{0} \circ \tilde{\pi}_{1} \circ \widetilde{\kappa}_{2} \circ D F \circ \kappa_{2}(w) \\
& =\tilde{\pi}_{0} \circ \tilde{\pi}_{1} \circ D F \circ \kappa_{2}(w)=\tilde{\pi}_{0} \circ F \circ \pi_{1} \circ \kappa_{2}(w)=C(\eta),
\end{aligned}
$$

and $\phi$ defines a single-valued map $\phi: M \rightarrow \widetilde{M}$. If $p \in M$, and $U$ is a non-vanishing vector field $U \in \mathfrak{X}(B)$ defined in a neighborhood $B \subset M$ of $p$, then

$$
\phi(x)=\tilde{\pi}_{0} \circ F \circ U(x), \quad x \in B
$$


and $\phi$ is smooth near $p$. To prove (ii), let $\xi \in T_{p} M \backslash\{0\}$, and let $U$ be a non-vanishing vector field defined near $p$ such that $U(p)=\xi$. Starting from $D \phi(\xi)=D\left(\widetilde{\pi}_{0} \circ F \circ U\right)(\xi)$, a similar calculation used to prove that map $C$ is constant shows that $D \phi(\xi)=F(\xi)$.

Lemma 3.3. If $F: T M \backslash\{0\} \rightarrow T \widetilde{M} \backslash\{0\}$ is a diffeomorphism, and $F=$ $\left.(D \phi)\right|_{T M \backslash\{0\}}$ for a smooth map $\phi: M \rightarrow \widetilde{M}$, then $\phi$ is a diffeomorphism.

Proof. Since $F$ is a diffeomorphism, we have $\operatorname{dim} M=\operatorname{dim} \widetilde{M}$, and by the inverse function theorem, $\phi$ is a local diffeomorphism. If $\xi \in T T \widetilde{M} \backslash\{0\} \cap T(T \widetilde{M} \backslash$ $\{0\})$, then there exists a $\zeta \in T(T M \backslash\{0\})$, such that $\xi=D F(\zeta)$. If $\zeta=\gamma^{\prime}(0)$ for a curve $\gamma:(-\varepsilon, \varepsilon) \rightarrow T M \backslash\{0\}$, equation (5) yields $0 \neq D \widetilde{\pi}_{0}(\xi)=D \phi \circ D \widetilde{\pi}_{0}(\zeta)$. Hence $\zeta \in T T M \backslash\{0\} \cap T(T M \backslash\{0\})$, so $D F(\zeta)=\widetilde{\kappa}_{2} \circ D F \circ \kappa_{2}(\zeta)$, and

$$
\kappa_{2} \circ D\left(F^{-1}\right) \circ \widetilde{\kappa}_{2}(\xi)=D\left(F^{-1}\right)(\xi) .
$$

By Lemma 3.2, there is a smooth map $\rho: \widetilde{M} \rightarrow M$ such that $F^{-1}=\left.D \rho\right|_{T \widetilde{M} \backslash\{0\}}$. Since $\rho \circ \phi=\left.i d\right|_{M}$ and $\phi \circ \rho=\left.\mathrm{id}\right|_{\widetilde{M}}$, it follows that $\phi$ is a diffeomorphism.

\section{Sprays}

The motivation for studying sprays is that they provide a unified framework for studying geodesics for Riemannian metrics, Finsler metrics, and non-linear connections. See [6], [24], [25]. Following [4], [5] we next define a spray on an iterated tangent bundle $T^{r} M$.

Definition 4.1. Let $r \geq 0$. Then a spray on $T^{r} M$ is a vector field $S \in$ $\mathfrak{X}\left(T^{r+1} M \backslash\{0\}\right)$ such that $\kappa_{r+2} \circ S=S$ and $\left[S, \mathbb{C}_{r+1}\right]=S$, where $\mathbb{C}_{r} \in \mathfrak{X}\left(T^{r} M\right)$, $r \geq 1$ is the Liouville vector field defined by

$$
\mathbb{C}_{r}(\xi)=\left.\partial_{t}(\xi+t \xi)\right|_{t=0}, \quad \xi \in T^{r} M .
$$

If $(x, y, X, Y)$ are local coordinates for $T^{r+2} M$ then a spray $S$ can be written as

$$
S(x, y)=\left(x, y, y,-2 G^{i}(x, y)\right)=y^{i} \frac{\partial}{\partial x^{i}}-2 G^{i}(x, y) \frac{\partial}{\partial y^{i}}
$$

for locally defined component functions $G^{i}: T^{r+1} M \backslash\{0\} \rightarrow \mathbb{R}$ that are positively 2-homogeneous. That is,

$$
G^{i}(\lambda y)=\lambda^{2} G^{i}(y), \quad \lambda>0 .
$$

A curve $c: I \rightarrow T^{r} M$ is regular if $c^{\prime}(t) \in T^{r+1} M \backslash\{0\}$ for all $t \in I$. That is, curve $c$ is regular if and only if its projection $\pi_{T^{r} M \rightarrow M} \circ c: I \rightarrow M$ is regular. 
Definition 4.2. If $S$ is a spray on $T^{r} M$ for $r \geq 0$, a regular curve $c: I \rightarrow T^{r} M$ is a geodesic if

$$
c^{\prime \prime}=S \circ c^{\prime}
$$

That is, a regular curve $c$ is a geodesic of spray $S$ if and only if $c^{\prime}$ is an integral curve of $S$. Conversely, suppose that $\gamma: I \rightarrow T^{r+1} M \backslash\{0\}$ is an integral curve of $S$, whence $\gamma^{\prime}=S \circ \gamma$. Since $\kappa_{r+2} \circ S=S$, there is a geodesic $c: I \rightarrow T^{r} M$, $c=\pi_{r} \circ \gamma$ such that $\gamma=c^{\prime}$.

Any geodesic $c: I \rightarrow T^{r} M$ of a spray $S$ is uniquely determined by $c^{\prime}\left(t_{0}\right)$ for one $t_{0} \in I$. The geodesic flow of a spray $S$ is defined as the flow of $S$ as a vector field, and a spray is complete if $S$ is complete as a vector field.

If $S$ is locally written as in equation (13) and $c(t)=\left(x^{i}(t)\right)$, then $c$ is a geodesic if and only if

$$
\ddot{x}^{i}(t)+2 G^{i} \circ c^{\prime}(t)=0 .
$$

4.1. Jacobi fields. We define Jacobi fields for a spray using the complete lift following [4], [5]. See also [16], [20], [30].

Definition 4.3. The complete lift of a spray $S$ on $M$ is the spray $S^{c} \in$ $\mathfrak{X}(T T M \backslash\{0\})$ on $T M$ given by

$$
S^{c}=D \kappa_{2} \circ \kappa_{3} \circ D S \circ \kappa_{2} .
$$

Suppose that $S$ is locally given by equation (13). Then $S^{c}$ is locally given by

$$
\begin{aligned}
S^{c} & =\left(x, y, X, Y, X, Y,-2 A^{i}(x, y, X, Y),-2 B^{i}(x, y, X, Y)\right) \\
& =X^{i} \frac{\partial}{\partial x^{i}}+Y^{i} \frac{\partial}{\partial y^{i}}-2 A^{i}(x, y, X, Y) \frac{\partial}{\partial X^{i}}-2 B^{i}(x, y, X, Y) \frac{\partial}{\partial Y^{i}}
\end{aligned}
$$

where $A^{i}$ and $B^{i}$ are vertical and complete lifts of functions $G^{i}$ [5],

$$
\begin{aligned}
& A^{i}(x, y, X, Y)=G^{i}(x, X), \\
& B^{i}(x, y, X, Y)=\frac{\partial G^{i}}{\partial x^{a}}(x, X) y^{a}+\frac{\partial G^{i}}{\partial y^{a}}(x, X) Y^{a} .
\end{aligned}
$$

Spray $S^{c}$ is complete if and only if spray $S$ is complete.

Definition 4.4. Suppose $S$ is a spray on $M$. A Jacobi field for $S$ is a geodesic $J: I \rightarrow T M$ of $S^{c}$.

If $J: I \rightarrow T M$ is a Jacobi field for $S$, then curve $c: I \rightarrow M, c=\pi_{0} \circ J$ is a geodesic for $S$ and we say that $J$ is a Jacobi field along $c$. Next we show that Definition 4.4 coincides with the usual characterization of Jacobi fields in terms of geodesic variations. For proofs and discussions, see [4], [5]. 
Definition 4.5. Suppose $S$ is a spray on $M$, and $c: I \rightarrow M$ is a geodesic for $S$. Then a geodesic variation of $c$ is a smooth map $V: I \times(-\varepsilon, \varepsilon) \rightarrow M$ such that

(i) $V(t, 0)=c(t)$ for all $t \in I$,

(ii) $t \mapsto V(t, s)$ is a geodesic for all $s \in(-\varepsilon, \varepsilon)$.

Suppose that $I$ is a closed interval. Then we say that a curve $J: I \rightarrow T M$ is a Jacobi field if we can extend $J$ into a Jacobi field defined on an open interval. Similarly, a map $V: I \times(-\varepsilon, \varepsilon) \rightarrow M$ is a geodesic variation if there is a geodesic variation $V^{*}: I^{*} \times\left(-\varepsilon^{*}, \varepsilon^{*}\right) \rightarrow M$ such that $V=V^{*}$ on the common domain of $V$ and $V^{*}$ and $I \subset I^{*}$.

Proposition 4.6. Let $S$ be a spray on $M$, let $J: I \rightarrow T M$ be a curve, where $I$ is open or closed, and let $c: I \rightarrow M$ be the curve $c=\pi_{0} \circ J$.

(i) If $J$ can be written as

$$
J(t)=\left.\partial_{s} V(t, s)\right|_{s=0}, \quad t \in I
$$

for a geodesic variation $V: I \times(-\varepsilon, \varepsilon) \rightarrow M$, then $J$ is a Jacobi field along $c$.

(ii) If $I$ is compact and $J$ is a Jacobi field along $c$, then there exists a geodesic variation $V: I \times(-\varepsilon, \varepsilon) \rightarrow M$ such that equation (15) holds.

Remark 4.7 (Zero Jacobi field). If $c: I \rightarrow M$ is a geodesic for a spray $S$, then the zero Jacobi field along $c$ is the Jacobi field $J: I \rightarrow T M$ that is locally induced by the constant geodesic variation $V(t, s)=c(t)$. Globally,

$$
J(t)=D \pi_{0} \circ \mathbb{C}_{1} \circ c^{\prime}(t), \quad t \in I .
$$

If zeroes of a Jacobi fields converge, then the Jacobi field is a zero Jacobi field.

\section{Maps that preserve structure}

Throughout this section we assume that $S$ and $\widetilde{S}$ are sprays on manifolds $M$ and $\widetilde{M}$, respectively. We proceed by studying maps that preserve (i) integral curves, (ii) geodesics, and (iii) Jacobi fields. In Section 7.1 we will also study maps between Riemann manifolds that preserve inner products. 
5.1. Maps that preserve integral curves. We say that a map

$$
F: T M \backslash\{0\} \rightarrow T \widetilde{M} \backslash\{0\}
$$

preserves integral curves if $F \circ \gamma: I \rightarrow T \widetilde{M} \backslash\{0\}$ is an integral curve of $\widetilde{S}$ whenever $\gamma: I \rightarrow T M \backslash\{0\}$ is an integral curve of $S$. When such a map $F$ exists, we say that sprays $S$ and $\widetilde{S}$ are conjugate. Condition (iii) in the next proposition shows that this corresponds to the usual definition of geodesic conjugacy in Riemann geometry [8], [27], [2].

Proposition 5.1. Suppose $F$ is a smooth map $F: T M \backslash\{0\} \rightarrow T \widetilde{M} \backslash\{0\}$. Then the following conditions are equivalent:

(i) $F$ preserves integral curves.

(ii) $\widetilde{S} \circ F=D F \circ S$ on $T M \backslash\{0\}$.

(iii) If $\Phi_{t}$ and $\widetilde{\Phi}_{t}$ are geodesic flows of $S$ and $\widetilde{S}$, respectively, then the following diagram commutes:

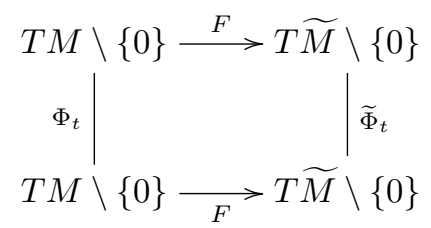

5.2. Maps that preserve geodesics. We say that a map

$$
\phi: M \rightarrow \widetilde{M}
$$

is a totally geodesic map if $\phi \circ c: I \rightarrow \widetilde{M}$ is a geodesic for $\widetilde{S}$ whenever $c: I \rightarrow M$ is a geodesic for $S[13$, Chapter 6$]$.

In Definition 4.2, we assume that geodesics are regular curves. If $\phi$ is a totally geodesic map, we can therefore restrict $D \phi$ to a map $D \phi: T M \backslash\{0\} \rightarrow T \widetilde{M} \backslash\{0\}$. Hence every totally geodesic map $\phi$ is an immersion, and if $\operatorname{dim} M=\operatorname{dim} \widetilde{M}$, then $\phi$ is also a local diffeomorphism. The definition of a totally geodesic map does not depend on derivatives of $\phi$. However, if $\phi: M \rightarrow \widetilde{M}$ is a homeomorphism, it follows that $\phi$ is a diffeomorphism [3].

Proposition 5.2. Suppose $\phi: M \rightarrow \widetilde{M}$ is a smooth immersion. Then $\phi$ is a totally geodesic map if and only if restriction $D \phi: T M \backslash\{0\} \rightarrow T \widetilde{M} \backslash\{0\}$ preserves integral curves. 
5.3. Maps that preserve Jacobi fields. We say that a map

$$
F: T M \backslash\{0\} \rightarrow T \widetilde{M} \backslash\{0\}
$$

preserves Jacobi fields if for any Jacobi field $J: I \rightarrow T M \backslash\{0\}$ without zeroes,

$$
\widetilde{J}(t)=F \circ J(t), \quad t \in I
$$

is a Jacobi field $\widetilde{J}: I \rightarrow T \widetilde{M} \backslash\{0\}$ without zeroes.

In the above definition, we only apply $F$ to Jacobi fields without zeroes. The next proposition shows that we can still map Jacobi fields with isolated zeroes.

Proposition 5.3. Suppose $\widetilde{S}$ is complete, $\operatorname{dim} M \geq 2$, and $F$ is a map $F: T M \backslash\{0\} \rightarrow T \widetilde{M} \backslash\{0\}$ that preserves Jacobi fields. If $J: \mathbb{R} \rightarrow T M$ is a Jacobi field for $S$ that is not identically zero, then there exists a Jacobi field $\widetilde{J}: \mathbb{R} \rightarrow T \widetilde{M}$ for $\widetilde{S}$ such that

$$
\widetilde{J}^{\prime}(t)=D F \circ J^{\prime}(t), \quad t \in \mathbb{R} \backslash Z,
$$

where $Z=\{t \in \mathbb{R}: J(t)=0\}$.

The proof of Proposition 5.3 is slightly technical and is given in Appendix A. The idea of the proof is to approximate a Jacobi field $J$ with an isolated zero by a variation of Jacobi fields without zeroes (see Lemma A.1). Then $F$ maps each non-zero Jacobi field in the variation into a non-zero Jacobi field, and a continuity argument shows that there exists a Jacobi field $\widetilde{J}$ as in equation (17).

Proposition 5.4. Suppose that map $F: T M \backslash\{0\} \rightarrow T \widetilde{M} \backslash\{0\}$ preserves integral curves, and suppose that $J: I \rightarrow T M$ is a Jacobi field for $S$. Then curve $\widetilde{J}: I \rightarrow T \widetilde{M}$,

$$
\widetilde{J}^{\prime}(t)=\widetilde{\kappa}_{2} \circ D F \circ \kappa_{2} \circ J^{\prime}(t), \quad t \in I
$$

is a Jacobi field for $\widetilde{S}$.

Proof. Equation (11) shows that curve $\widetilde{J}^{\prime}: I \rightarrow T T \widetilde{M} \backslash\{0\}$ is smooth. Proposition 5.1 and equations (11), (12) and (14) imply that

$$
\widetilde{S}^{c} \circ\left(\widetilde{\kappa}_{2} \circ D F \circ \kappa_{2}\right)=D\left(\widetilde{\kappa}_{2} \circ D F \circ \kappa_{2}\right) \circ S^{c} \quad \text { on } T T M \backslash\{0\},
$$

and $\widetilde{\kappa}_{2} \circ D F \circ \kappa_{2}$ maps integral curves of $S^{c}$ into integral curves of $\widetilde{S}^{c}$.

The next proposition is analogous to Proposition 5.2.

Proposition 5.5. Suppose $\phi: M \rightarrow \widetilde{M}$ is a smooth immersion. Then $\phi$ is a totally geodesic map if and only if restriction $D \phi: T M \backslash\{0\} \rightarrow T \widetilde{M} \backslash\{0\}$ preserves Jacobi fields. 
Proof. If $\phi$ is totally geodesic, then Propositions 5.2 and 5.4 imply that $D \phi$ preserves Jacobi fields. For the converse direction, suppose that $D \phi$ preserves Jacobi fields and $c: I \rightarrow M$ is a geodesic for $S$. Then $c^{\prime}$ is a Jacobi field for $S$, so $(D \phi) \circ c^{\prime}$ is a Jacobi field for $\widetilde{S}$, and $\widetilde{c}=\widetilde{\pi}_{0} \circ(\phi \circ c)^{\prime}=\phi \circ c$ is a geodesic $\widetilde{c}: I \rightarrow \widetilde{M}$ for $\widetilde{S}$.

\section{Descending maps for sprays}

In this section we prove Theorem 6.2, which gives sufficient conditions for a map $F: T M \backslash\{0\} \rightarrow T M \backslash\{0\}$ to descend to a totally geodesic map between two sprays. To formulate the assumptions in Theorem 6.2 we need the concept of a trapping hypersurface. This term is adapted from the concept of a non-trapping manifold with boundary.

Definition 6.1. Suppose $S$ is a spray on a manifold $M$. A hypersurface $\Sigma \subset M$ is a trapping hypersurface for $S$ if for any $y \in T M \backslash\{0\}$ there exists a geodesic $c: I \rightarrow M$ such that $c^{\prime}(0)=y$ and $c(t) \in \Sigma$ for some $t \in I$.

The existence of a trapping hypersurface $\Sigma$ imposes a global restriction on the behavior of geodesics. Namely, every geodesic has to intersect $\Sigma$. An interpretation is that if geodesics describe propagation of light, then the whole manifold is visible from the trapping hypersurface.

One way to construct a spray with a trapping hypersurfaces one can start with two sprays on a manifold $B$ with boundary $\partial B$. Using a smooth double one can glue together two copies of $B$ by identifying their boundary points. This gives a smooth manifold $M$ without boundary that contains two copies of the interior of $B$ and one copy of boundary $\partial B$. See [12], [15], or [19]. Assuming that the two sprays are nontrapping (see [10] for the Riemann case), and assuming that they satisfy suitable compatibility conditions on the boundary, one can glue together the sprays into a spray on $M$ such that boundary $\partial B \subset M$ is a trapping hypersurface. For example, any great circle on the 2 -sphere with the induced Euclidean metric is a trapping hypersurface.

Theorem 6.2. Suppose $S$ and $\widetilde{S}$ are complete sprays on a manifold $M$ with $\operatorname{dim} M \geq 2$. Furthermore, suppose that there exists a smooth map $F: T M \backslash\{0\} \rightarrow$ $T M \backslash\{0\}$ and a trapping hypersurface $\Sigma \subset M$ for $S$ such that

(i) F maps Jacobi fields for $S$ into Jacobi fields for $\widetilde{S}$ (see Section 5.3), 
(ii) for all $p \in \Sigma$,

$$
\begin{aligned}
S(y) & =\widetilde{S}(y), & & y \in T_{p} M \backslash\{0\}, \\
D F(\xi) & =\xi, & & \xi \in T\left(T_{p} M \backslash\{0\}\right) .
\end{aligned}
$$

Then there exists a smooth map $\phi: M \rightarrow M$ such that

(i) $F=\left.(D \phi)\right|_{T M \backslash\{0\}}$,

(ii) $\phi$ is a totally geodesic map (that maps geodesics for $S$ into geodesics for $\widetilde{S}$ ).

What is more, if $F$ is a diffeomorphism, then $\phi$ is a diffeomorphism.

In the proof below, Subcase B also proves Subcase A. However, Subcase A is included as it illustrates the main argument with minimal technical detail.

Proof. The proof is divided into two steps:

Step 1: Map F maps integral curves of $S$ into integral curves of $\widetilde{S}$.

Let $c^{\prime}: \mathbb{R} \rightarrow T M \backslash\{0\}$ be an integral curve of $S$, where $c$ is a geodesic $c: \mathbb{R} \rightarrow M$ of $S$. Then $c^{\prime}$ is a non-zero Jacobi field for $S$, and by assumption $6.2, J=F \circ c^{\prime}$, $J: \mathbb{R} \rightarrow T M \backslash\{0\}$ is a Jacobi field of $\widetilde{S}$ without zeroes. Since $\Sigma$ is trapping, there exists a $t_{0} \in \mathbb{R}$ such that $c\left(t_{0}\right) \in \Sigma$. By equations (5) and (19), we have $J^{\prime}\left(t_{0}\right)=c^{\prime \prime}\left(t_{0}\right)$. If $\eta: \mathbb{R} \rightarrow T M \backslash\{0\}$ is the integral curve of $\widetilde{S}$ determined by $\eta\left(t_{0}\right)=c^{\prime}\left(t_{0}\right)$, then $\eta^{\prime}\left(t_{0}\right)=\widetilde{S} \circ \eta\left(t_{0}\right)=J^{\prime}\left(t_{0}\right)$ by equation (18). Thus Jacobi fields $\eta$ and $J$ coincide and $J$ is an integral curve of $\widetilde{S}$.

Step 2: If $\xi \in T T M \backslash\{0\} \cap T(T M \backslash\{0\})$ we claim that

$$
D F(\xi)=\kappa_{2} \circ D F \circ \kappa_{2}(\xi) .
$$

If equation (20) holds, Theorem 3.1 implies that $F=\left.(D \phi)\right|_{T M \backslash\{0\}}$ for a map $\phi: M \rightarrow M$, whence assumption 6.2 and Proposition 5.5 imply that $\phi$ is totally geodesic. (Alternatively, one can use Step 1 and Proposition 5.2.) The last claim follows by Theorem 3.1 .

To prove equation (20), let $J: \mathbb{R} \rightarrow T M$ be the Jacobi field with $J^{\prime}(0)=\xi$, and let $c: \mathbb{R} \rightarrow M$ be the geodesic $c=\pi_{0} \circ J$. Since $\Sigma$ is a trapping hypersurface, there is a $t_{0} \in \mathbb{R}$ such that $c\left(t_{0}\right) \in \Sigma$.

Subcase $A: J\left(t_{0}\right) \neq 0$.

Propositions 5.3 and 5.4 imply that there exist Jacobi fields $J_{1}, J_{2}: \mathbb{R} \rightarrow T M$ for $\widetilde{S}$ such that

$$
\begin{array}{ll}
J_{1}^{\prime}(t)=D F \circ J^{\prime}(t), & \text { when } t \in \mathbb{R} \text { and } J(t) \neq 0, \\
J_{2}^{\prime}(t)=\kappa_{2} \circ D F \circ \kappa_{2} \circ J^{\prime}(t), & \text { when } t \in \mathbb{R} .
\end{array}
$$


Since $J\left(t_{0}\right) \neq 0$, we have

$$
J^{\prime}\left(t_{0}\right) \in T\left(T_{c\left(t_{0}\right)} M \backslash\{0\}\right)
$$

and since $t \mapsto J(t)$ is regular, we also have

$$
\kappa_{2} \circ J^{\prime}\left(t_{0}\right) \in T\left(T_{c\left(t_{0}\right)} M \backslash\{0\}\right) .
$$

Since $c\left(t_{0}\right) \in \Sigma$, equation (19) implies that $J^{\prime}\left(t_{0}\right)=J_{1}^{\prime}\left(t_{0}\right)=J_{2}^{\prime}\left(t_{0}\right)$. Hence $J_{1}=J_{2}$. Since $J(0) \neq 0$, it follows that

$$
D F(\xi)=J_{1}^{\prime}(0)=J_{2}^{\prime}(0)=\kappa_{2} \circ D F \circ \kappa_{2}(\xi) .
$$

Subcase B: $J\left(t_{0}\right)$ arbitrary.

Let $j: \mathbb{R} \times(-\varepsilon, \varepsilon) \rightarrow T M$, be the map

$$
j(t, s)=J(t)+s c^{\prime}(t), \quad(t, s) \in \mathbb{R} \times(-\varepsilon, \varepsilon) .
$$

Now $j(\cdot, s)$ is a Jacobi field (with only isolated zeroes) for all $s \in(-\varepsilon, \varepsilon)$. If $s \in(-\varepsilon, \varepsilon) \backslash\{0\}$, Propositions 5.3 and 5.4 imply that there exist Jacobi fields $j_{1}(\cdot, s), j_{2}(\cdot, s): \mathbb{R} \rightarrow T M$ for $\widetilde{S}$ such that

$$
\begin{array}{ll}
\partial_{t} j_{1}(t, s)=D F \circ \partial_{t} j(t, s), & \text { when } t \in \mathbb{R} \text { and } j(t, s) \neq 0, \\
\partial_{t} j_{2}(t, s)=\kappa_{2} \circ D F \circ \kappa_{2} \circ \partial_{t} j(t, s), & \text { when } t \in \mathbb{R} .
\end{array}
$$

Let $\varepsilon>0$ be such that $j\left(t_{0}, s\right) \neq 0$ and $j(0, s) \neq 0$ for all $s \in(-\varepsilon, \varepsilon) \backslash\{0\}$. Then

$$
\partial_{t} j\left(t_{0}, s\right) \in T\left(T_{c\left(t_{0}\right)} M \backslash\{0\}\right), \quad s \in(-\varepsilon, \varepsilon) \backslash\{0\},
$$

and since $t \mapsto j(t, s)$ is regular, we also have

$$
\kappa_{2} \circ \partial_{t} j\left(t_{0}, s\right) \in T\left(T_{c\left(t_{0}\right)} M \backslash\{0\}\right), \quad s \in(-\varepsilon, \varepsilon) \backslash\{0\} .
$$

Since $c\left(t_{0}\right) \in \Sigma$, equation (19) implies that $\partial_{t} j\left(t_{0}, s\right)=\partial_{t} j_{1}\left(t_{0}, s\right)=\partial_{t} j_{2}\left(t_{0}, s\right)$ for all $s \in(-\varepsilon, \varepsilon) \backslash\{0\}$, so $j_{1}(\cdot, s)=j_{2}(\cdot, s)$ for all $s \in(-\varepsilon, \varepsilon) \backslash\{0\}$. Let $\Xi$ be the smooth curve $\Xi:(-\varepsilon, \varepsilon) \rightarrow T T M \backslash\{0\}$,

$$
\Xi(s)=\left.\partial_{t} j(t, s)\right|_{t=0}, \quad s \in(-\varepsilon, \varepsilon) .
$$

Then

$$
D F \circ \Xi(s)=\kappa_{2} \circ D F \circ \kappa_{2} \circ \Xi(s), \quad s \in(-\varepsilon, \varepsilon) \backslash\{0\},
$$

and equation (20) follows since both sides of equation (21) are continuous for $s \in(-\varepsilon, \varepsilon)$ and since $\Xi(0)=\xi$. 


\section{Descending maps and isometries}

In this section we specialize Theorem 6.2 to the case when sprays $S$ and $\widetilde{S}$ are geodesic sprays of Riemann metrics. As a result we obtain Theorem 7.3, which gives sufficient conditions for two Riemann metrics to be isometric. It is not clear whether Theorem 7.3 also hold for Finsler metrics. However, the present proof uses that parallel transport is norm-preserving for Riemann metrics. This result generalize to Berwald metrics, but not to arbitrary Finsler metrics [26].

The geodesic spray of a (positive definite) Riemann metric $g$ is the spray with spray coefficients

$$
G^{i}(x, y)=\frac{1}{2} \Gamma_{a b}^{i}(x) y^{a} y^{b},
$$

where $\Gamma_{j k}^{i}$ are the Christoffel symbols associated with $g$.

Suppose $c: I \rightarrow M$ is a geodesic for a Riemann metric $g$ and $y \in T_{c(t)} M$ for some $t \in I$. Then there exists a unique curve $V: I \rightarrow T M$ such that (i) $\pi_{0} \circ V=c$, (ii) $V(t)=y$, and (iii) $\nabla V=0$, where $\nabla V$ is covariant derivative induced by $g$. We say that $V: I \rightarrow T M$ is the parallel transport of $y$ along $c$ and write $V(s)=$ $P_{t \rightarrow s}(c)(y)$ for $s \in I$. Thus $P_{t \rightarrow s}(c)$ is a linear map $P_{t \rightarrow s}(c): T_{c(t)} M \rightarrow T_{c(s)} M$. If $\phi: M \rightarrow \widetilde{M}$ is a totally geodesic map between Riemann manifolds and $c: I \rightarrow M$ is a geodesic, then $\phi$ commutes with the parallel transport, so that [28]

$$
(D \phi)\left(P_{t \rightarrow s}(c)(y)\right)=\widetilde{P}_{t \rightarrow s}(\phi \circ c)(D \phi(y)), \quad t, s \in I, y \in T_{c(t)} M .
$$

7.1. Isometric Riemann metrics. Suppose $\phi: M \rightarrow \widetilde{M}$ is a map and $g$ and $\widetilde{g}$ are Riemann metrics on $M$ and $\widetilde{M}$, respectively. Then $\phi$ is an isometry if for all $p \in M$,

$$
g(y, y)=\widetilde{g}(D \phi(y), D \phi(y)), \quad y \in T_{p} M .
$$

Every isometry is a totally geodesic map [1]. To prove Theorem 7.3, we will need the following converse result.

Proposition 7.1. Suppose $M$ and $\widetilde{M}$ are manifolds with Riemann metrics $g$ and $\widetilde{g}$, respectively. If $M$ is connected, $\phi$ is a totally geodesic map $\phi: M \rightarrow \widetilde{M}$, and equation (23) holds for one $p \in M$, then $\phi$ is an isometry.

Proof. For an open-closed argument, let

$$
A=\left\{q \in M: g(y, y)=\widetilde{g}(D \phi(y), D \phi(y)) \text { for } y \in T_{q} M\right\} .
$$

By continuity, $A$ is closed, and by assumption, $A$ is non-empty. To see that $A$ is open, let $q \in A$, and let $U \subset M$ be a normal coordinate neighborhood around $q$. 
If $r \in U$, then there exists a geodesic $c:[0,1] \rightarrow M$ such that $c(0)=q$ and $c(1)=r$. Then $\phi \circ c:[0,1] \rightarrow \widetilde{M}$ is also a geodesic. Using that parallel transport preserves Riemann norms, equation (22), and that $q \in A$, it follows that $r \in A$. Thus $A$ is open, and $M=A$.

The next proposition shows that a Riemann metric is essentially determined by its spray. This is a slight generalization of Lemma 1 on page 242 in [13].

Proposition 7.2. Suppose $g$ and $\widetilde{g}$ are Riemann metrics on a connected manifold $M$. If $g$ and $\widetilde{g}$ have the same geodesic spray and $g=\widetilde{g}$ on $T_{p} M$ for one $p \in M$, then $g=\widetilde{g}$.

Proof. This follows by taking $M=\widetilde{M}$ and $\phi=$ id in Proposition 7.1.

Theorem 7.3. Suppose $M$ is a smooth connected manifold $M$ with $\operatorname{dim} M \geq 2$ and $F$ is a smooth map $F: T M \backslash\{0\} \rightarrow T M \backslash\{0\}$. Furthermore, suppose that $g$ and $\widetilde{g}$ are complete Riemann metrics on $M$ such that $g$ has a trapping hypersurface $\Sigma \subset M$, and

(i) F maps Jacobi fields for $g$ into Jacobi fields for $\widetilde{g}$ (see Section 5.3),

(ii) for all $p \in \Sigma$,

$$
\begin{aligned}
S(y) & =\widetilde{S}(y), & & y \in T_{p} M \backslash\{0\}, \\
D F(\xi) & =\xi, & & \xi \in T\left(T_{p} M \backslash\{0\}\right),
\end{aligned}
$$

where $S$ and $\widetilde{S}$ are geodesic sprays induced by $g$ and $\widetilde{g}$, respectively,

(iii) for one $p \in M$,

$$
g(y, y)=\widetilde{g}(F(y), F(y)), \quad y \in T_{p} M \backslash\{0\} .
$$

Then there exists a smooth map $\phi: M \rightarrow M$ such that

(i) $F=\left.(D \phi)\right|_{T M \backslash\{0\}}$,

(ii) $\phi$ is an isometry (from $g$ to $\widetilde{g}$ ).

What is more, if $F$ is a diffeomorphism, then $\phi$ is a diffeomorphism.

Proof. This follows from Theorem 6.2 and Proposition 7.1. 


\section{Appendix A. Proof of Proposition 5.3}

For an outline of the proof below, see Section 5.3

Proof of Proposition 5.3. We can find a $t_{0} \in \mathbb{R}$ such that $J$ restricts to a Jacobi field $J: I_{0} \rightarrow T M \backslash\{0\}$ without zeroes where $I_{0} \subset \mathbb{R}$ is an neighborhood of $t_{0}$. Then $\widetilde{J}=F \circ J$ defines a Jacobi field $\widetilde{J}: I_{0} \rightarrow T \widetilde{M} \backslash\{0\}$ without zeroes. Since $\widetilde{S}$ is complete, $\widetilde{S}^{c}$ is complete [4], and Jacobi field $\widetilde{J}$ extends into a Jacobi field $\widetilde{J}: \mathbb{R} \rightarrow T \widetilde{M}$. For an open-closed argument, let $A=A_{e} \cup A_{0}$, where

$$
\begin{aligned}
& A_{e}=\left\{t \in \mathbb{R}: J(t) \neq 0 \text { and } \widetilde{J}^{\prime}(t)=D F \circ J^{\prime}(t)\right\} \\
& A_{0}=\left\{t \in \mathbb{R}: J(t)=0 \text { and }(t-\varepsilon, t) \cup(t, t+\varepsilon) \subset A_{e} \text { for some } \varepsilon>0\right\} .
\end{aligned}
$$

Set $A$ is non-empty since $I_{0} \subset A_{e}$. To see that $A$ is open, let us first note that $A_{e}$ is open since $F$ maps Jacobi fields without zeroes to Jacobi fields without zeroes and Jacobi fields are uniquely determined by their tangent at one point. Also, if $t \in A_{0}$, then $t$ has a neighborhood $N \subset \mathbb{R}$ such that $N \backslash\{t\} \subset A_{e}$.

To see that $A$ is closed, let $t_{i} \in A$ be a sequence such that $t_{i} \rightarrow \tau$ for some $\tau \in \mathbb{R}$. Let us show that $\tau \in A$. By Remark 4.7, we may assume that all $t_{i} \in A_{e}$. If $J(\tau) \neq 0$, then $\tau \in A_{e}$ by continuity. If $J(\tau)=0$, we show that $\tau \in A_{0}$. This is straightforward to check using uniqueness if an arbitrary neighborhood of $t$ contains $t_{i}$ :s on both sides of $\tau$. Let us assume that $t_{i}<\tau$ for all $i \geq 1$. (The case $t_{i}>\tau$ is analogous.)

Let $j: I \times(-\varepsilon, \varepsilon) \rightarrow T M$ be the map obtained by applying Lemma A.1 below to $J$. Then $\tau \in I$ and $j(t, s) \neq 0$ on for $(t, s) \neq(\tau, 0)$. Let $\widetilde{j}$ be the map

$$
\begin{aligned}
\widetilde{j}:(I \times(-\varepsilon, \varepsilon)) \backslash\{(\tau, 0)\} & \rightarrow T \widetilde{M} \backslash\{0\} \\
\widetilde{j}(t, s) & =F \circ j(t, s) .
\end{aligned}
$$

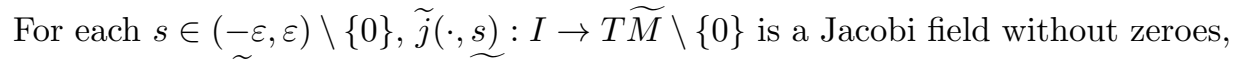
and for $s=0, \widetilde{j}(\cdot, 0): I_{ \pm} \rightarrow T \widetilde{M} \backslash\{0\}$ are Jacobi fields without zeroes, where

$$
I_{+}=\{t \in I: t>\tau\}, \quad I_{-}=\{t \in I: t<\tau\} .
$$

We know that $\widetilde{J}=\widetilde{j}(\cdot, 0)$ on $I_{-}$, and $\tau \in A_{0}$ follows if $\widetilde{J}=\widetilde{j}(\cdot, 0)$ on $I_{+}$. If $\widetilde{\Phi}_{t}^{c}$ is the flow of $\widetilde{S}^{c}$, and $t_{-} \in I_{-}$, then we have

$$
\begin{aligned}
\widetilde{j}\left(t_{+}, 0\right) & =\lim _{s \rightarrow 0} \widetilde{j}\left(t_{+}, s\right)=\lim _{s \rightarrow 0} \widetilde{\pi}_{1} \circ \widetilde{\Phi}_{t_{+}-t_{-}}^{c}\left(\partial_{t} \tilde{j}\left(t_{-}, s\right)\right) \\
& =\widetilde{\pi}_{1} \circ \widetilde{\Phi}_{t_{+}-t_{-}}^{c}\left(\widetilde{J}^{\prime}\left(t_{-}\right)\right)=\widetilde{J}\left(t_{+}\right)
\end{aligned}
$$

for all $t_{+} \in I_{+}$. 
Lemma A.1. Suppose $\operatorname{dim} M \geq 2, J: \mathbb{R} \rightarrow T M$ is a Jacobi field for spray $S$, and $\tau \in \mathbb{R}$ is an isolated zero for $J$. Then $\tau$ has a neighborhood $I \subset \mathbb{R}$, and there exists a map $j: I \times(-\varepsilon, \varepsilon) \rightarrow T M$ such that

(i) $j(t, 0)=J(t)$ for $t \in I$,

(ii) $t \mapsto j(t, s), t \in I$, is a Jacobi field for all $s \in(-\varepsilon, \varepsilon)$,

(iii) $j(t, s) \neq 0$ if $(t, s) \neq(\tau, 0)$.

Proof. We may assume that $\tau=0$. Let $c$ be geodesic $c: \mathbb{R} \rightarrow M, c=\pi_{0} \circ J$. In local coordinates, we have $J^{\prime}(0)=(x(0), 0, \dot{x}(0), \dot{J}(0))$, and let $\xi \in T_{c(0)} M \backslash\{0\}$ be vector $\xi=(x(0), \dot{J}(0))$. Then there exists an auxiliary Riemann metric on $M$ such that $g(\xi, \xi)=1$, and since $\operatorname{dim} M \geq 2$, there exists a non-zero vector $v \in T_{c(0)} M \backslash\{0\}$ such that $g(v, \xi)=0$ and $g(v, v)=1$. Let $K$ be a Jacobi field $K: I \rightarrow T M$ determined by $K^{\prime}(0)=(x(0), v, \dot{x}(0), 0)$, and let $j$ be the map $j: I \times \mathbb{R} \rightarrow T M$ defined as

$$
j(t, s)=J(t)+s K(t), \quad(t, s) \in I \times \mathbb{R} .
$$

Now (i) and (ii) are clear. For (iii), let us shrink $I$ such that $c: I \rightarrow M$ is contained in the domain of coordinates $x^{i}$. Then $j$ has local expression $j(t, s)=$ $\left(x^{i}(t), j^{i}(t, s)\right)$, and

$$
j^{i}(t, s)=\xi^{i} t+v^{i} s+R^{i}(t, s), \quad(t, s) \in I \times \mathbb{R},
$$

with remainder terms $R^{i}(t, s)=o\left(\sqrt{t^{2}+s^{2}}\right)$. For curve $j_{0}: I \times \mathbb{R} \rightarrow T_{c(0)} M$,

$$
j_{0}(t, s)=\left(x^{i}(0), j^{i}(t, s)\right)
$$

the Cauchy-Schwarz inequality yields

$$
g\left(j_{0}(t, s), j_{0}(t, s)\right)=t^{2}+s^{2}+o\left(t^{2}+s^{2}\right), \quad(t, s) \in I \times \mathbb{R},
$$

and

(iii) follows since we can find $\varepsilon>0$ such that

$$
g\left(j_{0}(t, s), j_{0}(t, s)\right) \geq \frac{1}{2}\left(t^{2}+s^{2}\right)
$$

for all $(t, s) \in(-\varepsilon, \varepsilon)^{2}$. 


\section{References}

[1] R. Abraham and J.Marsden, Foundations of Mechanics, Perseus Books, 1978.

[2] M. Berger, A Panoramic View of Riemann Geometry, Springer, 2007.

[3] F. Brickell, On the differentiability of affine and projective transformations, Proc. Amer. Math. Soc. 164 (1965), 567-574.

[4] I. Buchtaru and M. Dahl, A complete lift for semisprays, Int. J. Geom. Methods Mod. Phys. 72 (2010), 267-287.

[5] I. Buchtaru and M. Dahl, A geometric space without conjugate points, Balkan J. Geom. Appl. 151 (2010), 17-40.

[6] I. Bucataru and R. Miron, Finsler-Lagrange Geometry, Applications to dynamical systems, Romanian Academy, 2007.

[7] J. Cheeger and D. Ebin, Comparison Theorems in Riemannian Geometry, American Mathematical Society, 2008.

[8] C. Croke, Rigidity Theorems in Riemannian Geometry, vol. 137 of Geometric Methods in Inverse Problems and PDE control (The IMA Volumes in Mathematics and its Applications), Springer, 2004, 47-72.

[9] A. DA Silva, Lectures on Symplectic Geometry, Springer, 2008.

[10] N. Dairbekov, Integral geometry problem for nontrapping manifolds, Inverse Problems 22, no. 2 (2006), 431-445.

[11] A. Haro, The primitive function of an exact symplectomorphism, Nonlinearity 13 (2000), $1483-1500$.

[12] M. HiRsch, Differential Topology, Springer, 1976.

[13] S. Kobayashi and K. Nomizu, Foundations of Differential Geometry, Vol. 1, Interscience publishers, 1963.

[14] R. Kulkarni, Curvature and metric, Ann. of Math. 912 (1970), 311-331.

[15] J. LEE, Introduction to Smooth Manifolds, Springer, 2006.

[16] A. LEWIs, The geometry of the maximum principle for affine connection control systems, preprint (2000), 1-52.

[17] P. Libermann and C.-M. Marle, Symplectic Geometry and Analytical Mechanics, Springer, 1987.

[18] M.-C. LiU, Affine maps on tangent bundles., Indiana Univ. Math. J. 237 (1974), 593-605.

[19] Y. матsumoto, An Introduction to Morse Theory, American Mathematical Society, 2002.

[20] P. Michor, The Jacobi flow., Rend. Sem. Mat. Univ. Pol. Torino 544 (1996), 365-372.

[21] R. Miron, D. Hrimiuc, H. Shimada and S. V. Sabau, The Geometry of Hamilton and Lagrange Spaces, Kluwer Academic Press, 2001.

[22] K. Nomizu and K. YAno, Some results related to the equivalence problem in Riemann geometry, Math. Z. 97 (1967), 29-37.

[23] L. Pestov and G. Uhlmann, Two dimensional compact simple Riemannian manifolds are boundary rigid, Ann. of Math. 161 (2005), 1089-1106.

[24] T. SAKAI, Riemannian Geometry, American Mathematical Society, 1996.

[25] Z. Shen, Differential Geometry of Spray and Finsler Spaces, Springer, 2001.

[26] Z. Shen, Lectures of Finsler geometry, World Scientific, 2001.

[27] G. Uhlmann, Travel time tomography, J. Korean Math. Soc. 384 (2001), 711-722.

[28] J. VILms, Totally geodesic maps, J. Differential Geometry 4 (1970), 73-79. 
250 I. Bucataru and M. F. Dahl : Descending maps between slashed tangent...

[29] A. Weinstein, Lectures on Symplectic Manifolds, American Mathematical Society, 1976.

[30] K. Yano and S. Ishinara, Tangent and Cotangent Bundles, Marcel Dekker Inc., 1973.

[31] S.-T. YAU, Curvature preserving diffeomorphisms, Ann. of Math. 100 (1974), 121-130.

IOAN BUCATARU

FACULTY OF MATHEMATICS

AL.I.CUZA UNIVERSITY

B-DUL CAROL 11

IASI 700506

ROMANIA

E-mail: bucataru@uaic.ro

URL: http://www.math.uaic.ro/ ${ }^{\sim}$ bucataru

MATIAS DAHL

DEPARTMENT OF MATHEMATICS

AND SYSTEMS ANALYSIS

AALTO UNIVERSITY

FI-00076 AALTO

FINLAND

E-mail: matias.dahl@tkk.fi

$U R L:$ http://math.tkk.fi/ ${ }^{\sim}$ dahl

(Received September 21, 2010; revised March 28, 2011) 